\title{
PERFIL CLÍNICO-EPIDEMIOLÓGICO DE INDIVÍDUOS COM HISTÓRICO DE TRAUMATISMO CRANIOENCEFÁLICO
}

\section{CLINICAL-EPIDEMIOLOGICAL PROFILE OF \\ INDIVIDUALS WITH TRAUMATIC BRAIN INJURY HISTORY}

\section{PERFIL CLÍNICO-EPIDEMIOLÓGICO DE INDIVIDUOS CON HISTORIAL DE TRAUMATISMO CRANEOENCEFÁLICO}

\author{
Jocinei Ferreira Constâncio ${ }^{1}$ \\ Adriana Alves Nery ${ }^{2}$ \\ Edilene Curvelo Hora Mota ${ }^{3}$ \\ Clarice Alves dos Santos ${ }^{4}$ \\ Maitana Carvalho Cardozo \\ Tatiane Oliveira de Souza Constâncio ${ }^{6}$
}

Como citar este artigo: Constâncio JF, Nery AA, Mota ECH, Santos CA, Cardozo MC, Constâncio TOS. Perfil clínico-epidemiológico de indivíduos com histórico de traumatismo cranioencefálico. Rev baiana enferm; 2018;32:e28235.

Objetivo: descrever o perfil clínico-epidemiológico dos indivíduos com histórico de Traumatismo Cranioencefálico atendidos em um hospital público do interior do estado da Bahia e conhecer as características clínico-epidemiológicas desses indivíduos segundo sexo e causas (violentas e não violentas). Método: estudo descritivo de abordagem quantitativa, exploratório, realizado com pacientes internados por Traumatismo Cranioencefálico. Os dados foram submetidos à análise bivariada, utilizando o Teste Qui-quadrado de Pearson. Resultado: compôs a amostra, um total de 1.140 prontuários de indivíduos com média de idade de 37,6 ( $\pm 20,5)$ anos, sendo a maioria do sexo masculino (81,9\%). Predominou, na casuística, indivíduos com traumatismo cranioencefálico leve (36\%), sendo as causas mais incidentes os acidentes de transportes envolvendo motocicleta (30,5\%), automóvel $(16,4 \%)$ e as quedas da própria altura (14,6\%). Conclusão: as principais vítimas de traumatismo cranioencefálico foram adultos jovens do sexo masculino e as principais causas foram os acidentes de transporte e as quedas.

Descritores: Traumatismos Cranioencefálicos. Lesão Encefálica Traumática. Epidemiologia. Perfil de Saúde. Morbidade.

\footnotetext{
Fisioterapeuta. Mestre em Ciências da Saúde. Professor Assistente do Departamento de Ciências Biológicas da Universidade Estadual do Sudoeste da Bahia. Jequié, Bahia, Brasil. jocinei@gmail.com

Enfermeira. Doutora em Enfermagem em Saúde Pública. Professora do Departamento de Saúde II e do Programa de Pós-graduação em Enfermagem e Saúde da Universidade Estadual do Sudoeste da Bahia. Jequié, Bahia, Brasil.

Enfermeira. Doutora em Enfermagem na Saúde do Adulto. Professora Associada IV do Departamento de Enfermagem e do Programa de Pós-graduação em Ciências da Saúde da Universidade Federal de Sergipe. Aracaju, Sergipe, Brasil.

Educadora física. Mestra em Saúde Coletiva. Professora Assistente do Departamento de Ciências Biológicas da Universidade Estadual do Sudoeste da Bahia. Jequié, Bahia, Brasil.

Enfermeira. Médica. Médica Assistente do Hospital Geral Prado Valadares. Jequié, Bahia, Brasil.

Enfermeira. Mestra em Ciências da Saúde. Jequié, Bahia, Brasil.
} 
Objective: to describe the clinical-epidemiological profile of individuals with traumatic brain injury history admitted to the public hospital of a city in the state of Babia, and learn their clinical-epidemiological characteristics according to gender and causes (violent and non-violent). Method: a descriptive and exploratory study with a quantitative approach was carried out with patients hospitalized due to traumatic brain injury. Data were submitted to bivariate analysis with the use of Pearson's chi-square test. Result: the sample was made up of 1,140 medical records of individuals with a mean age of 37.6 ( \pm 2.5 ) years, mostly men (81.9\%). In casuistry, there was a prevalence of individuals with minor traumatic brain injury (36\%), mostly caused by transport accidents involving motorcycles (30.5\%), cars (16.4\%) and falls from their own beight (14.6\%). Conclusion: the main victims of traumatic brain injury were young male adults and the main causes were transport accidents and falls.

Descriptors: Craniocerebral Trauma. Traumatic Brain Injuries. Epidemiology. Health Profile. Morbidity.

Objetivo: describir el perfil clínico-epidemiológico de individuos con historial de traumatismo craneoencefálico atendidos en hospital público del interior del estado de Babia y conocer sus características clínico-epidemiológicas según sexo y causas (violentas y no violentas). Método: estudio descriptivo de abordaje cuantitativo, exploratorio, realizado con pacientes internados por traumatismo craneoencefálico. Datos sometidos a análisis bivariado, utilizándose el Test de Chi-cuadrado de Pearson. Resultado: muestra constituida por 1.140 bistorias clinicas de individuos con media etaria de 37,6 ( $\pm 20,5)$ años, siendo la mayoría $(81,9 \%)$ de sexo masculino. Predominaron en la casuística los individuos con traumatismo craneoencefálico leve (36\%), resultando las causas más repetidas los accidentes de transportes involucrando motocicleta (30,5\%), automóvil (16,4\%) y caídas desde la propia altura (14,6\%). Conclusión: las principales víctimas de traumatismo craneoencefálico fueron adultos jóvenes de sexo masculino, y las principales causas fueron los accidentes de tránsito y las caídas.

Descriptores: Traumatismos Craneocerebrales. Lesionas Traumáticas del Encéfalo. Epidemiología. Perfil de Salud. Morbilidad.

\section{Introdução}

O Traumatismo Cranioencefálico (TCE) afeta milhões de pessoas em todo o mundo. É uma das principais causas de morbidade e mortalidade, especialmente em adultos jovens ${ }^{(1)}$.

Dados processados e disponibilizados pelo Departamento de Informática do SUS (DATA/ SUS) demonstram que, entre janeiro de 2008 e setembro de 2018, o Brasil atingiu um número de 1.090.258 internações por Traumatismos Intracranianos, predominantes nas regiões Sudeste, correspondendo a 42,5\% (463.396), e Nordeste do país, com 25,8\% (281.907) ${ }^{(2)}$.

Pesquisas revelam que o Traumatismo Cranioencefálico é a principal causa de morte e sequelas na faixa etária de 1 a 44 anos. A taxa de mortalidade do TCE grave está acima de 30\%. As principais sequelas provocadas pelo TCE grave são as neurológicas, comprometendo a qualidade de vida das pessoas acometidas ${ }^{(3)}$.

As causas mais comuns de TCE são os acidentes envolvendo veículos, especialmente em adolescentes e adultos jovens. As quedas são as responsáveis pelo segundo maior grupo de lesões e são mais comuns em crianças e idosos. Em alguns locais, as lesões provocadas por arma de fogo provocam mais TCE do que acidentes automobilísticos ${ }^{(3)}$.

Para a criação de estratégias que possam minimizar as causas e consequências do TCE numa população, é necessário conhecer todos os fatores envolvidos no processo. Nesse sentido, é de fundamental importância que os grupos mais vulneráveis, bem como os aspectos sociodemográficos e clínicos associados a esse tipo de trauma sejam identificados, para que ações de planejamento e prevenção sejam realizadas com maior resolutividade.

Nessa perspectiva, o presente estudo tem como objetivo descrever o perfil clínico-epidemiológico dos indivíduos com histórico de Traumatismo Cranioencefálico atendidos em um Hospital Público do interior da Bahia e conhecer as características clínico-epidemiológicas desses indivíduos segundo sexo e causas (violentas e não violentas). 


\section{Método}

Trata-se de um estudo epidemiológico, descritivo de abordagem quantitativa, exploratório, realizado no município de Jequié, Bahia, com os pacientes admitidos no Hospital Geral Prado Valadares (HGPV), com registro de TCE no prontuário. O HGPV é habilitado como Unidade de Assistência de Alta Complexidade em Traumatologia e Ortopedia. O público-alvo atendido na Instituição em questão é formado por usuários do Sistema Único de Saúde (SUS).

Os participantes do estudo foram os pacientes com histórico de internamento no HGPV por TCE e que atenderam aos critérios de inclusão: admissão por TCE de qualquer gravidade no período compreendido entre 2009 e 2014. Para identificação dos participantes do estudo, foi feito um levantamento no Serviço de Arquivo Médico Estatístico (SAME) dos pacientes admitidos na Emergência do HGPV, com diagnóstico de TCE, no período estudado.

O tempo de internação hospitalar foi registrado considerando-se os dias internados da admissão até a alta (ou óbito) e classificado em "breve", "normal" ou "prolongado" conforme critérios julgados pelo Ministério da Saúde, disponíveis em aplicativos do Sistema de Gerenciamento da Tabela de Procedimentos, Medicamentos, Órteses, Próteses e Materiais especiais (SIGTAP).

Para classificar o tempo de internação hospitalar em breve, normal ou prolongado, utilizou-se como parâmetro a média específica de dias para cada diagnóstico de TCE leve, moderado ou grave, ou do procedimento neurocirúrgico realizado, quando foi o caso. A média de dias considerada normal para cada caso foi estabelecida de acordo com o SIGTAP. Somente foi calculada a permanência dos pacientes com o escore de gravidade do TCE registrado e que não possuíam lesões associadas com potencial para interferir na média de dias estimada para cada caso.

O estudo foi realizado seguindo-se as orientações contidas na Resolução n. 466/2012, do Conselho Nacional de Saúde (CNS), que aborda os aspectos éticos da pesquisa envolvendo seres humanos. O projeto foi encaminhado, para análise, ao Comitê de Ética em Pesquisa da Universidade Estadual do Sudoeste da Bahia (CEP/ UESB), sendo aprovado pelo Parecer n. 961.356.

Após liberação para coleta de dados, foi feito um levantamento de todos os prontuários de pacientes com diagnóstico de TCE, com ou sem tratamento neurocirúrgico, nos quais os dados para caracterização da amostra foram colhidos.

Os pesquisadores elaboraram um formulário para o registro de informações sociodemográficas e clínicas (sexo, idade, tempo de internação hospitalar, lesões associadas, tratamento etc.) disponíveis nos prontuários. Esses dados foram posteriormente alimentados no banco de dados para análise.

A Escala de Coma de Glasgow (ECG) foi utilizada como referência para categorizar os pacientes em níveis de gravidade nomeadamente definidos como TCE Leve (ECG de 13 a 15), TCE Moderado (ECG de 9 a 12) e TCE Grave (ECG de 3 a 8$)^{(4)}$.

As causas foram dicotomizadas em violentas (trauma violento por arma de fogo ou branca, agressão física, espancamento) e não violentas (acidentes envolvendo veículos de transporte em geral, quedas e pancadas não intencionais).

Os dados foram tabulados e organizados no aplicativo "Planilhas do Google", transferidos para o Excel e, desse, para o Statistical Package for the Social Sciences (SPSS). Em seguida, foram submetidos à análise da estatística descritiva simples, por meio de cálculos das médias e desvio padrão para as variáveis quantitativas, e a apresentação das frequências absolutas e relativas das variáveis categóricas. Para estimar as proporções do TCE nos grupos por sexo (masculino e feminino) e por causas (violentas e não violentas), os dados foram submetidos à análise bivariada. Utilizou-se o Qui-quadrado de Pearson, com um nível de significância de 5\% (0,05).

\section{Resultados}

As características clínico-epidemiológicas dos 1.140 indivíduos que compuseram a população 
deste estudo estão apresentadas na Tabela 1. A média de dias de internação hospitalar, incluindo toda a amostra, foi de 11,0 $( \pm 15,7)$ dias, com mediana de 6,0. O mínimo de dias internado foi 0 (zero) e o máximo de 188 dias.

Tabela 1 - Características clínico-epidemiológicas dos indivíduos com histórico de Traumatismo Cranioencefálico admitidos no Hospital Geral Prado Valadares. Jequié, Bahia, Brasil - $2015(\mathrm{~N}=1140)$

(continua)

\begin{tabular}{l|l|l}
\hline \multirow{2}{*}{ Variável } & \multicolumn{2}{|c}{ Frequências } \\
\cline { 2 - 3 } & $\mathbf{n}$ & $\%$ \\
\hline
\end{tabular}

\section{Sexo}

Feminino

Masculino

\section{Grupo etário (taxa de resposta 96\%)}

$1-9$

$10-19$

\section{Causas do Traumatismo Cranioencefálico}

Acidentes de transporte envolvendo motocicleta

Acidentes de transporte envolvendo automóvel ou veículos

Acidentes de transporte envolvendo bicicleta

Trauma violento por arma de fogo

Trauma violento por arma branca

Trauma violento por espancamento / agressão física

Queda da própria altura

Queda de altura

Contusão e pancadas não intencionais

Trauma sem causa identificada

\section{Lesões associadas}

Sem registro $\quad 634$

Fratura de extremidades

Fratura de face

Politraumatismo sem registro de fratura e /ou luxação

Politraumatismo com fratura e/ou luxação

Fratura e/ou luxação da coluna vertebral

Escoriações e contusões sem registro de fratura

\section{Gravidade do Traumatismo Cranioencefálico}

Leve

Moderado

Grave

Sem registro

\section{Tratamento}


Tabela 1 - Características clínico-epidemiológicas dos indivíduos com histórico de Traumatismo Cranioencefálico admitidos no Hospital Geral Prado Valadares. Jequié, Bahia, Brasil - $2015(\mathrm{~N}=1140)$ (conclusão)

\begin{tabular}{l|c|c}
\hline \multirow{2}{*}{ Variável } & \multicolumn{2}{|c}{ Frequências } \\
\cline { 2 - 3 } Tempo de Internação Hospitalar & n & \% \\
Breve & 156 & 13,7 \\
Normal & 277 & 24,3 \\
Prolongada & 88 & 7,7 \\
Não calculada & 619 & 54,3 \\
Óbito Hospitalar & & \\
Não & 984 & 86,4 \\
Sim & 126 & 11,1 \\
Sem registro & 30 & 2,6 \\
\hline
\end{tabular}

Fonte: Elaboração própria.

Os dados da Tabela 2 mostram a relação entre as características clínico-epidemiológicas dos indivíduos e a história de TCE e suas causas violentas e não violentas. Foram verificados resultados estatisticamente significantes $(\mathrm{p} \leq 0,05)$ para as seguintes características: grupo etário $(\mathrm{p}=0,043)$, lesões associadas $(0,013)$, tratamento $(0,001)$ e permanência na instituição hospitalar $(0,012)$. Houve uma predominância da gravidade leve $(87,1 \%)$ e tratamento conservador $(89,2 \%)$ no TCE de causa não violenta.

Tabela 2 - Características clínico-epidemiológicas dos indivíduos com histórico de Traumatismo Cranioencefálico segundo causa (Não violenta x Violenta). Jequié, Bahia, Brasil - 2015

(continua)

\begin{tabular}{l|c|c|c|c|c}
\hline \multirow{2}{*}{ Variáveis } & \multicolumn{2}{|c|}{ Não Violenta } & \multicolumn{2}{c|}{ Violenta } & \multirow{2}{*}{ p-valor* } \\
\cline { 2 - 4 } & $\mathbf{n}$ & $\mathbf{\%}$ & $\mathbf{n}$ & $\mathbf{\%}$ & \\
\hline Sexo (Taxa de resposta 93,9\%) & & & & & \\
$\quad$ Feminino & 173 & 91,1 & 17 & 8,9 & 0,184 \\
Masculino & 772 & 87,6 & 109 & 12,4 & \\
Grupo etário (Taxa de resposta 92,8\%) & & & & & \\
$1-9$ & 40 & 97,6 & 1 & 2,4 & 0,043 \\
$10-19$ & 131 & 87,3 & 19 & 12,7 & \\
$20-29$ & 247 & 86,7 & 38 & 3,3 & \\
$30-39$ & 167 & 86,5 & 26 & 13,5 & \\
$40-59$ & 192 & 86,5 & 30 & 13,5 & \\
$60-74$ & 86 & 92,5 & 7 & 7,5 & \\
$\geq 75$ & 72 & 97,3 & 2 & 2,7 & \\
Lesões Associadas (Taxa de resposta 93,9\%) & & & & & \\
Sem Registro & 511 & 86,5 & 80 & 13,5 & 0,013 \\
Fratura de extremidades & 55 & 94,8 & 3 & 5,2 & \\
Fratura em face & 30 & 90,9 & 3 & 9,1 & \\
Politraumatismo sem registro de fratura e/ou & 105 & 89,0 & 13 & 11,0 & \\
luxação & & & & & \\
Politraumatismo com fratura e/ou luxação & 64 & 100,0 & - & - & \\
Fratura e/ou luxação da coluna vertebral & 16 & 100,0 & - & - & \\
Escoriações e contusões sem registro de fratura & 164 & 85,9 & 27 & 14,1 &
\end{tabular}


Tabela 2 - Características clínico-epidemiológicas dos indivíduos com histórico de Traumatismo Cranioencefálico segundo causa (Não violenta x Violenta). Jequié, Bahia, Brasil - 2015

(conclusão)

\begin{tabular}{|c|c|c|c|c|c|}
\hline \multirow{2}{*}{ Variáveis } & \multicolumn{2}{|c|}{ Não Violenta } & \multicolumn{2}{|c|}{ Violenta } & \multirow{2}{*}{ p-valor* } \\
\hline & $\mathbf{n}$ & $\%$ & $\mathbf{n}$ & $\%$ & \\
\hline \multicolumn{6}{|c|}{ Gravidade do Traumatismo Cranioencefálico (Taxa de resposta $70,0 \%$ ) } \\
\hline Leve & 338 & 87,1 & 50 & 12,9 & 0,235 \\
\hline Moderado & 172 & 91,5 & 16 & 8,5 & \\
\hline Grave & 193 & 86,5 & 30 & 13,5 & \\
\hline \multicolumn{6}{|c|}{ Tratamento (Taxa de resposta 90,7\%) } \\
\hline Conservador & 830 & 89,2 & 100 & 10,8 & 0,001 \\
\hline Cirúrgico & 81 & 77,9 & 23 & 22,1 & \\
\hline \multicolumn{6}{|c|}{ Tempo de Internação Hospitalar (Taxa de resposta 43,8\%) } \\
\hline Breve & 124 & 83,2 & 25 & 16,8 & 0,012 \\
\hline Normal & 236 & 89,4 & 28 & 10,6 & \\
\hline Prolongada & 67 & 77,0 & 20 & 23,0 & \\
\hline \multicolumn{6}{|c|}{ Óbito Hospitalar (Taxa de resposta 91,5\%) } \\
\hline Não & 820 & 88,6 & 106 & 11,4 & 0,894 \\
\hline Sim & 104 & 88,1 & 14 & 11,9 & \\
\hline
\end{tabular}

Fonte: Elaboração própria.

* Teste Qui-quadrado de Person com nível de significância de 5\% ( $<<0,05)$.

Nota: Sinal convencional utilizado:

- Dado numérico igual a zero não resultante de arredondamento.

A Tabela 3 mostra as características clínico-epidemiológicas dos indivíduos com histórico de TCE segundo o sexo, com diferenças proporcionais estatisticamente significantes $(\mathrm{p} \leq 0,05)$ para o grupo etário $(\mathrm{p}=0,000)$ e para as lesões associadas $(\mathrm{p}=0,040)$. A média de idade entre os indivíduos do sexo feminino foi de 43,9 anos $( \pm 25,5)$, enquanto no sexo masculino foi de 36,3 anos $( \pm 18,9)$. Não houve diferenças proporcionais estatisticamente significantes entre os sexos nas demais características analisadas $(\mathrm{p}>0,05)$.

Tabela 3 - Características clínico-epidemiológicas dos indivíduos com histórico de Traumatismo Cranioencefálico segundo sexo. Jequié, Bahia, Brasil - 2015

(continua)

\begin{tabular}{|c|c|c|c|c|c|}
\hline \multirow{2}{*}{ Variáveis } & \multicolumn{2}{|c|}{ Masculino } & \multicolumn{2}{|c|}{ Feminino } & \multirow{2}{*}{ p-valor* } \\
\hline & $\mathbf{n}$ & $\%$ & $\mathbf{n}$ & $\%$ & \\
\hline \multicolumn{6}{|l|}{ Grupo etário (Taxa de resposta 98,7\%) } \\
\hline $1-9$ & 34 & 79,1 & 9 & 20,9 & 0,000 \\
\hline $10-19$ & 126 & 79,7 & 32 & 20,3 & \\
\hline $20-29$ & 261 & 87,9 & 36 & 12,1 & \\
\hline $30-39$ & 173 & 86,5 & 27 & 13,5 & \\
\hline $40-59$ & 207 & 85,9 & 34 & 14,1 & \\
\hline $60-74$ & 73 & 72,3 & 28 & 27,7 & \\
\hline$\geq 75$ & 50 & 58,1 & 36 & 41,9 & \\
\hline \multicolumn{6}{|l|}{ Lesões Associadas (Taxa de resposta 98,5\%) } \\
\hline Sem registro & 521 & 82,2 & 113 & 17,8 & 0,040 \\
\hline Fratura de extremidades & 40 & 67,8 & 19 & 32,2 & \\
\hline Fratura em face & 26 & 74,3 & 9 & 25,7 & \\
\hline Politraumatismo sem registro de fratura e /ou luxação & 107 & 83,6 & 21 & 16,4 & \\
\hline Politraumatismo com fratura e/ou luxação & 60 & 88,2 & 8 & 11,8 & \\
\hline \multicolumn{6}{|l|}{ Fratura e/ou luxação da coluna vertebral } \\
\hline Escoriações e contusões & 164 & 82,4 & 35 & 17,6 & \\
\hline
\end{tabular}


Tabela 3 - Características clínico-epidemiológicas dos indivíduos com histórico de Traumatismo Cranioencefálico segundo sexo. Jequié, Bahia, Brasil - 2015

(conclusão)

\begin{tabular}{|c|c|c|c|c|c|}
\hline \multirow{2}{*}{ Variáveis } & \multicolumn{2}{|c|}{ Masculino } & \multicolumn{2}{|c|}{ Feminino } & \multirow{2}{*}{ p-valor* } \\
\hline & $\mathbf{n}$ & $\%$ & $\mathbf{n}$ & $\%$ & \\
\hline \multicolumn{6}{|c|}{ Gravidade do Traumatismo Cranioencefálico (Taxa de resposta 73,7\%) } \\
\hline Leve & 331 & 80,7 & 79 & 19,3 & 0.350 \\
\hline Moderado & 160 & 82,5 & 34 & 17,5 & \\
\hline Grave & 202 & 85,2 & 35 & 14,8 & \\
\hline \multicolumn{6}{|c|}{ Tratamento (Taxa de resposta $96,1 \%$ ) } \\
\hline Conservador & 800 & 81,2 & 185 & 18,8 & 0,260 \\
\hline Cirúrgico & 95 & 85,6 & 16 & 14,4 & \\
\hline \multicolumn{6}{|c|}{ Tempo de Internação Hospitalar (Taxa de resposta $45,7 \%$ ) } \\
\hline Breve & 132 & 84,6 & 24 & 15,4 & 0,485 \\
\hline Normal & 224 & 80,9 & 53 & 19,1 & \\
\hline Prolongada & 75 & 85,2 & 13 & 14,8 & \\
\hline \multicolumn{6}{|c|}{ Óbito Hospitalar } \\
\hline Não & 803 & 81,9 & 177 & 18,1 & 0,635 \\
\hline Sim & 101 & 80,2 & 25 & 19,8 & \\
\hline
\end{tabular}

Fonte: Elaboração própria.

* Teste Qui-quadrado de Person com nível de significância de 5\% ( $<<0,05)$.

\section{Discussão}

O reconhecimento do TCE como um problema de saúde pública é justificado em diversos estudos pela sua relação com elevados níveis de morbimortalidade e por afetar, principalmente, o grupo etário de indivíduos ativos da população ${ }^{(3,5-7)}$. No atual estudo, houve maior prevalência de casos de TCE do sexo masculino e do grupo etário de 20 a 29 anos (26,4\%), tal como se verifica em muitos estudos ${ }^{(5-6,8-9)}$.

Os acidentes envolvendo veículos de transporte estão entre as principais causas de TCE, entre os quais destacam-se aqueles envolvendo motocicletas $^{(8-12)}$, tal como demonstrado neste estudo cuja prevalência correspondeu a 30,5\% dos casos encontrados. No entanto, algumas pesquisas apontam as quedas como a primeira causa de TCE, principalmente a queda da própria altura ${ }^{(13-14)}$. Os elevados índices desse tipo de queda são relacionados à faixa etária dos indivíduos: idosos ou crianças. No caso dos idosos, decorre das alterações fisiológicas a que estão submetidos com o passar do tempo ${ }^{(13)}$. Nas crianças, esses índices são justificados pelas características do desenvolvimento, bem como pelos comportamentos próprios da infância ${ }^{(14)}$.

De acordo com o Sistema de Informações sobre Mortalidade (SIM), no período de 2008 a 2016 houve um total de 101.593 mortes por acidente de motocicleta no Brasil, com predomínio nas regiões Nordeste e Sudeste do país ${ }^{(2)}$. O crescente uso das motocicletas como meio de transporte pode ser atrelado ao baixo custo desse veículo e à rapidez e agilidade que ele proporciona em um trânsito cada vez mais congestionado.

Em estudo realizado no ano de 2011, no mesmo município do presente estudo, foi relatado que a falta de qualidade do transporte público municipal tem levado a população a procurar a motocicleta como meio de transporte mais cômodo e mais barato para se locomover. Esta situação, associada ao aumento do índice de desemprego, fez com que muitos indivíduos adquirissem a moto para trabalhar como mototáxi na cidade, mas este serviço não possui regulamentação nem fiscalização adequada no município em questão ${ }^{(12)}$. 
O nível de consciência dos indivíduos após o trauma influencia significativamente no prognóstico do paciente com diagnóstico de TCE ${ }^{(11)}$. Por este motivo, é importante a classificação de gravidade de acordo com a ECG, método de avaliação neurológica que qualifica o nível de consciência dos indivíduos após o trauma e serve também como indicador na melhora da função cerebral $^{(11,14)}$.

O TCE classificado como leve foi o mais encontrado (36\%) entre os indivíduos estudados, enquanto que 20,8\% representaram os TCE notificados como graves e $17 \%$, os moderados. Da mesma forma, foi observada, em outros estudos, maior prevalência do TCE leve quanto à gravidade ${ }^{8,11,13-14}$. É importante ressaltar que 26,2\% dos prontuários consultados não apresentavam o registro da ECG, semelhante a outros estudos em que os prontuários consultados não tiveram a gravidade inicial do trauma devidamente $\operatorname{anotada}^{(11,14)}$.

O tipo de tratamento predominante foi $\mathrm{O}$ conservador, acompanhando o maior índice de ocorrência de traumas de gravidade leve, da mesma forma como ocorrido em estudo que apresentou um índice de 77,18\% de tratamento conservador das pessoas internadas, juntamente com a prevalência de traumatismo de gravidade leve ${ }^{(13)}$. Já a média do tempo de internação encontrada foi de 11,5 dias, valor maior do que o encontrado em alguns estudos, que obtiveram uma média 7,2 e 8,7 dias ${ }^{(13,15)}$.

As lesões associadas ao TCE dependem dos mecanismos e da energia cinética imposta ao indivíduo. Sobre essa questão, há relatos de ocorrência em maior ou igual frequência dos traumas (abdômen, tórax, face e membros) e de otorragia e, em menor incidência, das fraturas sistêmicas ${ }^{(13)}$. Neste estudo, as lesões mais encontradas foram as escoriações e contusões sem registro de fratura e o politraumatismo sem registro de fratura e/ou luxação.

A taxa de mortalidade no presente estudo foi de $11,1 \%$ com $66,3 \%$ desses casos correspondendo a indivíduos com registros de ECG grave. A esse respeito, os estudos têm apresentado uma relação inversamente proporcional entre o número de mortes e a pontuação inicial na ECG, isto é, quanto menor o valor do escore na ECG, analisada logo após o evento do trauma, maior o índice de mortalidade entre os indivíduos ${ }^{(8)}$. Um número expressivo de prontuários sem o registro da ECG foi identificado no presente estudo $(26,2 \%)$, todavia, tal como se observa em outros estudos, o TCE leve sempre prepondera sobre as outras gravidades e sobre as taxas de óbito $^{(8-9,12,14)}$.

Neste estudo, foram considerados traumas "não violentos" aqueles que não envolveram causa externa intencional, tais como o uso de arma branca, arma de fogo ou qualquer outro meio de agressão física. No tocante ao grupo de indivíduos com histórico de TCE por causas "não violentas", este estudo encontrou a predominância de traumatismos de gravidade leve $(87,1 \%)$, com tratamento conservador $(89,4 \%)$ e sem desfecho com óbito (88,6\%). Tais resultados corroboram os obtidos na maioria dos estudos consultados $^{(8-9,10-14)}$.

Estudos mostram predominância de indivíduos do sexo masculino entre as vítimas do TCE ${ }^{(5-6,8-9)}$. Da mesma forma, eles são responsáveis pelos maiores índices de internação e taxas de mortalidade ${ }^{(6)}$. Esse fato foi também verificado no presente estudo, apesar de não serem detectadas diferenças em ambos os sexos quanto à predominância do tipo de gravidade, tratamento, tempo de internação hospitalar e óbito.

As limitações encontradas no presente estudo dizem respeito à ausência de importantes registros sobre os pacientes, entre eles a ECG na admissão, lesões associadas ao trauma e o tempo de internação hospitalar. A falta dessas informações implica em um conhecimento menos detalhado do perfil clínico-epidemiológico dos casos que envolvem TCE.

\section{Conclusão}

Os resultados deste estudo permitiram descrever o perfil clínico-epidemiológico dos indivíduos com histórico de TCE e conhecer as características clínico-epidemiológicas dos indivíduos com histórico de Traumatismo Cranioencefálico, 
segundo sexo e causas (violentas e não violentas). Os resultados possibilitaram identificar, na população estudada, que o TCE predomina entre os indivíduos jovens do sexo masculino, com média de idade de 37,69 anos e com gravidade classificada como leve. Os acidentes envolvendo motocicletas foram as principais causas de TCE e o tratamento mais adotado foi o conservador. A média de internação hospitalar foi de 11,05 dias e a taxa de óbito hospitalar de 11,1\%. As principais lesões associadas ao TCE encontradas foram escoriações e contusões sem registro de fratura e o politraumatismo sem registro de fratura e/ou luxação.

Todos esses resultados mostraram o predomínio de causas "não violentas" que podem ser evitadas, principalmente por medidas preventivas educacionais e de fiscalização, quais sejam campanhas de prevenção nas estradas, nas escolas, veiculação de propagandas de prevenção pelos meios de comunicação de massa (televisão, rádios, jornais, revistas, mídias sociais etc.).

\section{Colaborações:}

1. concepção, projeto, análise e interpretação dos dados: Jocinei Ferreira Constâncio, Adriana Alves Nery, Edilene Curvelo Hora Mota e Clarice Alves dos Santos;

2. redação do artigo e revisão crítica relevante do conteúdo intelectual: Jocinei Ferreira Constâncio, Maitana Carvalho Cardozo e Tatiane Oliveira de Souza Constâncio;

3. aprovação final da versão a ser publicada: Jocinei Ferreira Constâncio e Tatiane Oliveira de Souza Constâncio.

\section{Referências}

1. Klein AM, Howell K, Vogler J, Grill E, Straube A, Bender A. Rehabilitation Outcome of Unconscious Traumatic Brain Injury Patients. J Neurotrauma [Internet]. 2013 [cited 2016 Aug 10];30(17):1476-83. Available from: Doi: 10.1089/neu.2012.2735

2. Brasil. Departamento de Informática do SUS - DATASUS. Informações de Saúde [Internet]. Brasília; 2018 [cited 2018 Nov 23]. Available from: http://tabnet.datasus.gov.br/cgi/tabcgi.exe?sih/ cnv/niuf.def

3. Gaudêncio TG, Leão GM. A epidemiologia do traumatismo crânio-encefálico: um levantamento bibliográfico no Brasil. Rev Neurocienc [Internet]. 2013 [cited 2016 Aug 30];21(3):427-34. Available from: Doi: 10.4181/RNC.2013.21.814.8p

4. Teasdale G, Jennett B. Assessment of coma and impaired consciousness. A practical scale. Lancet [Internet]. 1974 [cited 2016 Aug 30];2(7872):81-4. Available from: https://www.ncbi.nlm.nih.gov/ pubmed/4136544

5. Vieira RCA, Hora EC, Oliveira DV, Vaez AC. Relevamiento epidemiológico de los accidentes motociclísticos atendidos en un Centro de Referencia de Trauma de Sergipe. Rev Esc Enferm USP [Internet]. 2011 [cited 2018 Sep 1];45(6):135963. Available from: Doi: http://dx.doi.org/10.1590/ S0080-62342011000600012

6. Dantas IEF, Oliveira TT, Machado Neto CD. Epidemiologia do traumatismo crânio encefálico (TCE) no Nordeste no ano de 2012. REBES [Internet]. 2014 jan-mar [cited 2018 Sep 10];4(1):1823. Available from: https://www.gvaa.com.br/ revista/index.php/REBES/article/view/2573/1985

7. Walder B, Haller G, Rebetez MML, Delhumeau C, Bottequin E, Schoettker P, et al. Severe traumatic brain injury in a high-income country: an epidemiological study. J Neurotrauma [Internet]. 2013 Dec [cited 2016 Sep 10];30(23):1934-42. Available from: Doi: 10.1089/neu.2013.2955

8. Lopes RD, Costa PP, Carvalho FT. Perfil epidemiológico dos pacientes acometidos por trauma cranioencefálico assistidos em um hospital público de Teresina. Rev Bras Neurol Psiquiatr [Internet]. 2013 [cited 2017 Aug 28];7(3):80-90. Available from: https://www.revneuropsiq.com. $\mathrm{br} / \mathrm{rbnp} /$ article/view/27/17

9. Moura JC, Rangel BLR, Creôncio SCE, Pernambuco JRB. Perfil clínico-epidemiológico de traumatismo cranioencefálico do Hospital de Urgências e Traumas no município de Petrolina, Estado de Pernambuco. Arq Bras Neurocir [Internet]. 2011 [cited 2018 Aug 27];30(3):99-104. Available from: http://files.bvs.br/upload/S/0103-5355/2011/ v30n3/a2709.pdf

10. Nnadi MON, Bankole OB, Fente BG. Motorcycle-related traumatic brain injuries: helmet use ante treatment outcome. Neurosci J [Internet]. 
2015 [cited 2018 Sep 3];2015:1-6. Available from: Doi: http://dx.doi.org/10.1155/2015/696787

11. Barbosa IL, Andrade LM, Caetano JA, Lima MA, Vieira LJES, Lira SVG. Fatores desencadeantes ao trauma crânio-encefálico em um hospital de emergência municipal. Rev baiana saúde pública [Internet]. 2010 [cited 2018 Sep 3];34(2):240-53. Available from: http://files.bvs.br/ upload/S/0100-0233/2010/v34n2/a1795.pdf

12. Gomes IO, Reis LA, Mascarenhas CHM. Perfil dos pacientes acometidos por trauma cranioenfálico admitidos em um hospital público do município de Jequié na Bahia. Rev Saúde Com [Internet]. 2011 [cited 2018 Aug];7(1):14-22. Available from: http://www.uesb.br/revista/rsc/v7/v7n1a02.pdf

13. Maia BG, Paula FRP, Cotta GD, Cota MAL, Públio PG, Oliveira H, et al. Perfil clínico-epidemiológico das ocorrências de traumatismo cranioencefálico. Rev Neurocienc [internet]. 2013 [cited 2018
Sep];21(1):43-52. Available from: Doi: 10.4181/ RNC.2013.21.786.10p

14. Santos F, Casagranda LP, Lange C, Farias JC, Pereira PM, Jardim VMR, et al. Traumatismo Cranioencefálico: causas e perfil das vítimas atendidas no pronto-socorro de Pelotas/Rio Grande do Sul, Brasil. REME rev min enferm [Internet]. 2013 [cited 2018 Aug 30];17(4):882-7. Available from: http://www.reme.org.br/artigo/ detalhes/893

15. Ramos EMS, Silva MKB, Siqueira GR, Vieira RAG, França WLC. Aspectos epidemiológicos dos traumatismos cranioencefálicos atendidos no Hospital Regional do Agreste de Pernambuco de 2006 a 2007. RBPS [Internet]. 2010 [cited 2018 Sep 2];23(1):4-10. Availablefrom: Doi: http://dx.doi.org/10.5020/1164 Recebido: 27 de setembro de 2018 Aprovado: 11 de novembro de 2018

Publicado: 27 de dezembro de 2018

A Revista Baiana de Enfermagem utiliza a Licença Creative Commons - Atribuição-NãoComercial 4.0 Internacional. https://creativecommons.org/licenses/by-nc/4.0/ Este artigo é de acesso aberto distribuído sob os termos da Licença Creative Commons (CC BY-NC). Esta licença permite que outros remixem, adaptem e criem a partir do seu trabalho para fins não comerciais. Embora os novos trabalhos tenham de lhe atribuir o devido crédito e não possam ser usados para fins comerciais, os usuários não têm de licenciar esses trabalhos derivados sob os mesmos termos. 